\title{
Avaliação da resistência de larvas de Contracaecum sp. (nematoda: anisakidae) à ação da citronela, Cymbopogon sp., in natura, administrada como tratamento da anisakiose em coelhos infectados experimentalmente
}

\author{
Evaluation of the resistence of the Contracaecum sp. larvae, (nematoda: \\ anisakidae), to the action of the citronella, Cymbopogon sp., in natura, \\ administered as treatment of anisakiosis in experimentally infected rabbits
}

Christiano Henrique da Silva Justino, * Leone Duarte Medeiros, * Maria Luzinete Alves Vanzeler, ${ }^{* *}$ Luciano Antunes Barros ${ }^{\star \star *}$

\section{Resumo}

A avaliação da ação larvicida da citronela, Cymbopogon sp., in natura foi feita através da administração da planta ad libitum a coelhos, Oryctolagus cuniculi, experimentalmente infectados com larvas de Contracaecum sp. Com intervalos regulares de 24 horas após o início da administração da planta, foram realizadas eutanásias e necropsias dos animais infectados, analisando-se a eficácia através de parâmetros como número e viabilidade de larvas recuperadas. A citronela in natura apresentou baixa ação larvicida sobre Contracaecum sp.

Palavras-chave: Citronela, Cymbopogon sp., Contracaecum sp., Anisakidae, coelhos infectados experimentalmente.

\begin{abstract}
The evaluation of the larvicide action of the citronella, Cymbopogon sp., in natura was done through the feeding of the citronella grass ad libitum to rabbits, Oryctolagus cuniculi, experimentally infected by Contracaecum sp. larvae. By regular intervals of 24 hours after the beginning of the experiment, the infected animals went submitted to eutanasy and necropsy exams to analyse the action of the plant, using parameters as the number and viability of the recovered larvae. The citronella in natura produced low larvicide action over Contracaecum sp. experimentally.
\end{abstract}

Keywords: Citronella, Cymbopogon sp., Contracaecum sp., Anisakidae, rabbits experimentally infected.

\section{Introdução}

O consumo de pescado é uma prática comum em muitas populações humanas, desde a Antiguidade. Isso se deve principalmente ao alto valor nutricional desta fonte de alimento e à facilidade logística de sua captura para alguns povos. Porém, em algumas culturas pode-se observar o hábito de consumir o pescado cru ou malcozido, predispondo ao risco de contrair enfermidades parasitárias.

O estudo das zoonoses parasitárias transmitidas pelo consumo da carne de pescado torna-se fundamental para a saúde pública, uma vez que o homem pode assumir o papel de hospedeiro acidental, contraindo infecções parasitárias como a eustrongilidose, a anisaquiose, a difilobotriose, a fagicolose e outras, que podem levar a manifestações clínicas graves (Chieffi et al., 1990; Chieffi et al. 1992; Santos e Faro, 2005; Eduardo et al., 2005a,b; Emmel et al. 2006; Leite et al., 1989; Dias et al., 1992).

O despreparo dos profissionais de saúde e a falta de conhecimento mais aprofundado sobre este assunto são agravantes à situação de importância destas parasitoses.

Para o estado de Mato Grosso são relatadas prevalências altas do parasitismo por larvas de Contracaecum sp. e Eustrongylides sp., em peixes como a traíra (Hoplias malabaricus), piraputanga (Brycon microlepis), piranha (Serrassalmus marginatus), cachara (Pseudoplatystoma fasciatum), barbado (Pinirampus pirinampu), pintado

\footnotetext{
Laboratório de Parasitologia Veterinária, Departamento de Produção Animal, Faculdade de Agronomia e Medicina Veterinária, UFMT, Av. Fernando Corrêa s/n, Coxipó, Cuiabá, MT, CEP 78069-900, chrisjustino@hotmail.com

** Faculdade de Ciências Médicas FCM/UFMT, Av. Fernando Correa da Costa s/n Cuiabá, MT CEP 78069-900, vanzeler@terra.com.br

*** Laboratório de Doenças Parasitárias, Departamento de Saúde Coletiva Veterinária e Saúde Pública, Faculdade de Medicina Veterinária, UFF, Rua Vital Brazil Filho, 64, CEP 24230-340, Santa Rosa, Niterói, RJ, labarros@terra.com.br

A quem enviar correspondência. E-mail: labarros@terra.com.br
} 
(Pseudoplatystoma corruscans) e jaú (Paulicea luetkeni (Barros et al., 2006; Barros et al., 2007).

$\mathrm{O}$ crescente aumento na indústria do pescado, associado à pouca informação sobre métodos profiláticos e de tratamento para estas parasitoses, revelam a premente necessidade de buscar informações sobre a ocorrência destes parasitos em espécies de peixes destinadas ao consumo humano, assim como métodos seguros de diagnóstico e testes de eficácia de terapias voltadas para o controle de larvas em hospedeiros infectados.

\section{Material e métodos}

Duzentos e quarenta espécimes de traíras, Hoplias malabaricus (Bloch, 1794), foram capturadas com anzol no rio Mimoso, município de Santo Antônio de Leverger, localizado a $140 \mathrm{~km}$ de Cuiabá, estado de Mato Grosso, a $16^{\circ} 12^{\prime} 50^{\prime \prime}$ de latitude Sul, $55^{\circ} 48^{\prime} 29^{\prime \prime}$ de longitude Oeste. Após a captura, estes peixes foram acondicionados em caixas térmicas e transportados ao Laboratório de Parasitologia Veterinária da Universidade Federal de Mato Grosso (UFMT), onde foram medidos, pesados e necropsiados para a coleta de larvas de Contracaecum sp., segundo metodologia descrita por Amato et al. (1991). Os dados referentes às necropsias foram anotados em fichas individuais e as larvas encontradas foram mantidas em placas de Petri, com solução salina fisiológica $(0,65 \% \mathrm{NaCl})$ para serem avaliadas quanto à viabilidade em estereomicroscópio, utilizando como parâmetros a motilidade e a integridade estrutural. A identificação taxonômica foi realizada segundo Vicente et al. (1995).

A planta citronela, Cymbopogon sp., utilizada foi cultivada no setor de horticultura da Faculdade de Agronomia e Medicina Veterinária da UFMT, para ser fornecida in natura e ad libitum a modelos experimentais infectados com larvas de Contracaecum sp. Utilizou-se a citronela como única fonte de alimento durante o experimento, garantindo a maior ingestão possível da planta. Com o objetivo de minimizar riscos de alteração ou perda do(s) princípio(s) ativo(s) por evaporação e secagem, utilizando-se folhas verdes e recém-coIhidas.

Vinte e quatro coelhos (Oryctolagus cuniculi) machos adultos, da raça Nova Zelândia, provenientes do Biotério Central da UFMT, foram utilizados como modelos experimentais, subdivididos em seis grupos, contendo quatro animais cada, sendo utilizada a seguinte denominação para os grupos:

- Grupo Controle Geral (GCG): composto por animais não infectados, e alimentados com ração comercial para coelhos;

- Grupo Controle Citronela (GCC): composto por animais não infectados e alimentados apenas com citronela $a d$ libitum;

- Grupo Controle Parasitado (GCP): composto por animais infectados, com larvas de Contracaecum sp. e alimentados com ração comercial para coelhos; e

- Grupos Testes (GT1, GT2 e GT3): compostos por animais infectados com larvas de Contracaecum sp. e alimentados apenas com citronela ad libitum.

Os animais foram mantidos em gaiolas individuais, em ambiente refrigerado com temperatura e umidade relativa controladas, $24,1 \pm 5,5^{\circ} \mathrm{C}, 51 \pm 15 \%$.
Após a separação dos grupos foram selecionadas 560 larvas de Contracaecum sp. consideradas viáveis para infecção. Essas larvas foram subdivididas em 16 inóculos com 35 larvas/animal e administradas aos coelhos a serem infectados, seguindo a técnica descrita por Barros et al. (2004). Os animais não foram deixados em jejum préinfecção, para que a velocidade na taxa da passagem do inóculo pelo trato gastrintestinal fosse mantida dentro dos padrões de normalidade fisiológica, não interferindo desse modo na fixação das larvas à mucosa do trato gastrointestinal. Durante as primeiras 24 horas pós-infecção, os animais foram mantidos com ração e água ad libitum, observandose possíveis alterações comportamentais.

Decorridas as primeiras 24 horas, iniciou-se o fornecimento da planta aos grupos GT1, GT2, GT3 e GCC em substituição à ração comercial. Com intervalos regulares de 24 horas após o início da administração da planta, foi eutanasiado e necropsiado um coelho de cada grupo, para avaliar a ação da planta sobre as larvas nos Grupos Testes, bem como analisar possíveis alterações macroscópicas e microscópicas nos locais de fixação das larvas no GCP. Os animais do GCG foram mantidos vivos até o 70 dia após o início do experimento, a fim de avaliar eventuais interferências que pudessem representar risco à saúde dos mesmos. Os animais do GCC foram mantidos vivos até o 14 dia após o início do experimento, para avaliação de possíveis alterações nos parâmetros hematológicos e reações de toxicidade causados pelo consumo da planta, segundo os critérios recomendados por BRASIL (1996). A insensibilização e a eutanásia dos animais obedeceram às normas preconizadas por Brasil (2000) e a necropsia foi realizada segundo a técnica descrita por Feldman e Seely (1988).

Todas as larvas recuperadas durante a necropsia foram coletadas e mantidas em placas de Petri, contendo solução salina fisiológica $(0,8 \% \mathrm{NaCl})$. As larvas mortas foram fixadas com formalina $10 \%$, e posteriormente processadas conforme as técnicas histológicas descritas por Prophet (1992), sendo cortadas com espessura de $3 \mu \mathrm{m}$, por uso de micrótomo manual Microm ${ }^{\circledast}$ MOD. HM 325. A coloração foi realizada pela técnica de hematoxilina-eosina (Allen, 1992) e posteriormente o material foi examinado e microfotografado, com aumentos de 40X e 100X, em microscópio óptico Olympus ${ }^{\circledR}$ BX-41.

Os animais do GCP e GCC foram submetidos aos mesmos exames laboratoriais, utilizando-se os valores de referência apresentados por Mitruka e Rawnsley (1977).

Para a avaliação estatística da resistência das larvas de Contracaecum sp. à planta in natura, foi realizado o teste qui-quadrado, utilizando-se como ferramenta o programa estatístico Epi Info ${ }^{\mathrm{TM}}$ 6.0.

\section{Resultados}

Os dados clínicos apresentados pelo GCG foi considerado livre de doenças baseado no conjunto de achados dos exames clínicos (temperatura, frequência cardíaca, movimento respiratório) e complementares (volume globular, hemoglobina), que apresentaram resultados dentro dos padrões de normalidade.

Durante a execução da necropsia foram recuperadas 258 larvas no trato gastrointestinal, destas $7(2,71 \%)$ mortas não 
aderidas à mucosa, 197 (76,36\%) vivas aderidas e 54 $(20,93 \%)$ vivas não aderidas. Uma larva foi encontrada viva e aderida ao peritônio de um dos animais dos Grupos Testes (Tabelas 1 e 2) (Figura 1). Macroscopicamente, observou-se que as larvas aderidas à mucosa do intestino causaram

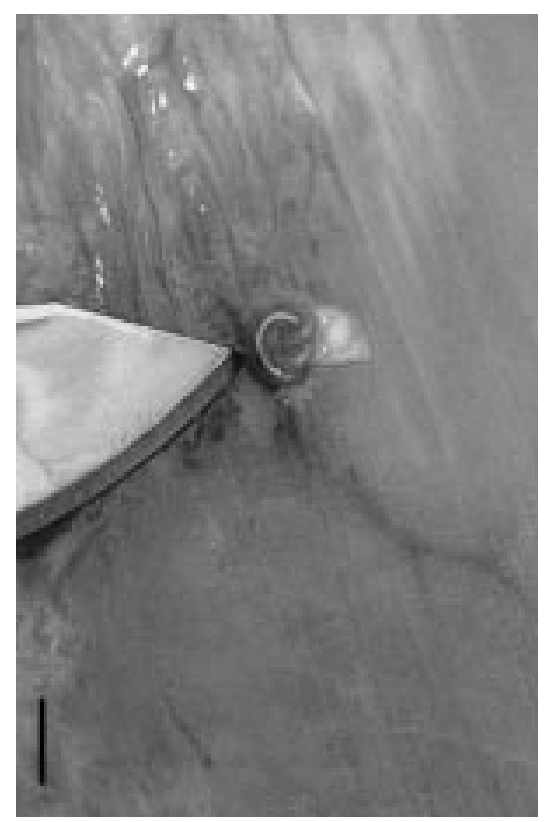

Figura 1: Macroscopia de uma larva de Contracaecum sp. aderida ao peritônio de coelho infectado experimentalmente, em exame 48 horas pós-infecção. (barra $=0,25 \mathrm{~cm}$ )

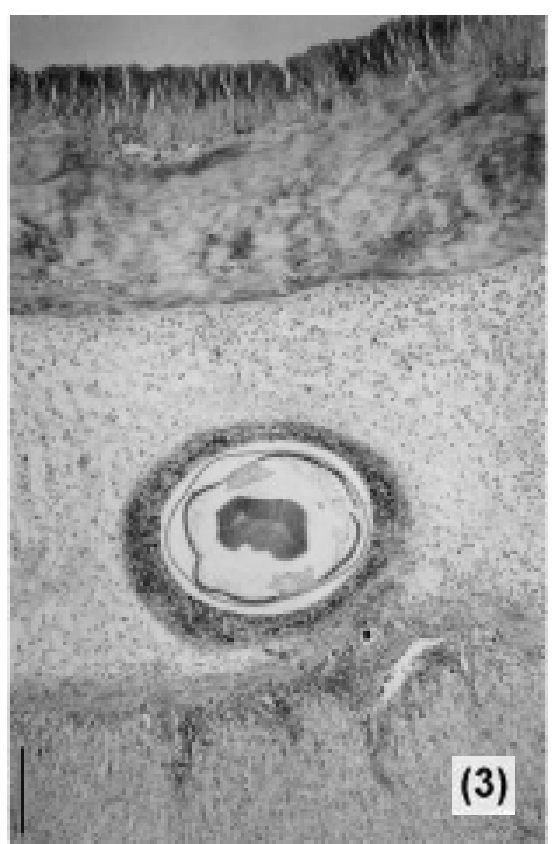

Figura 3: Corte histológico do estômago de coelho (Grupo Teste), infectado com larva de Contracaecum sp., circundada por reação inflamatória com predominância de eosinófilos e neutrófilos e espessamento de submucosa por edema e infiltrado polimorfonuclear, corado por Hematoxilina-Eosina. $($ barra $=2 \mu \mathrm{m})$ hiperemia local e lesão ulcerativa com bordos elevados. À microscopia óptica, foi observada reação inflamatória com predominância de eosinófilos e neutrófilos, além de espessamento de submucosa por edema e infiltrado polimorfo nuclear (Figuras 2 a 4).

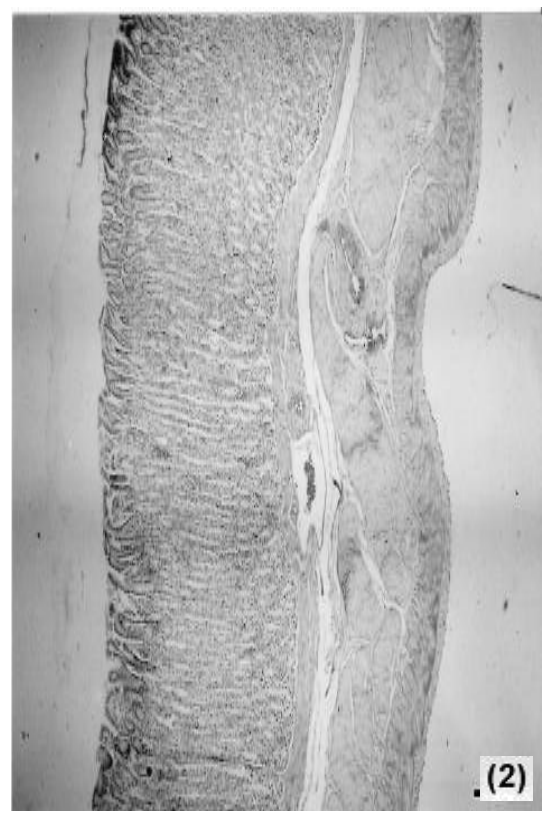

Figura 2: Corte histológico do estômago de coelho (Grupo Controle Geral), sem alterações histológicas, corado por HematoxilinaEosina. (barra $=2 \mu \mathrm{m}$ )

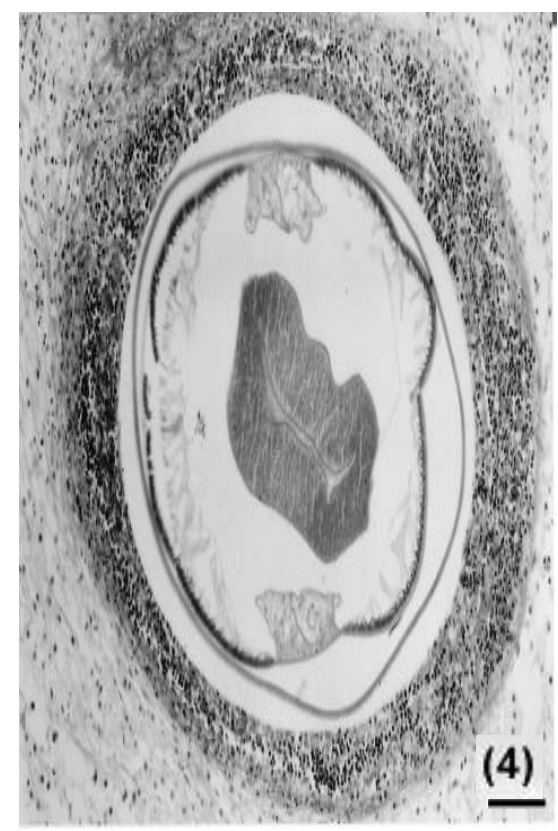

Figura 4: Corte histológico de estômago de coelho (Grupo Teste), infectado com de larva de Contracaecum sp. localizada na submucosa, circundada por reação inflamatória com predominância de eosinófilos e neutrófilos, corado por Hematoxilina-Eosina. (barra $=5 \mu \mathrm{m}$ ) 
Tabela 1: Larvas de Contracaecum sp. recuperadas no período pós-infecção de coelhos dos grupos teste (GT1, GT2 e GT3) e Grupo Controle Parasitado (GCP)

\begin{tabular}{cccccc}
\hline $\begin{array}{c}\text { Período pós } \\
\text { infecção (dias) }\end{array}$ & \multicolumn{5}{c}{ Grupos } \\
\hline $\mathbf{1}$ & GCP & GT1 $^{*}$ & GT2 & GT3 & Total \\
$\mathbf{2}$ & 28 & 11 & 13 & 13 & $\mathbf{6 5}$ \\
$\mathbf{3}$ & 10 & 23 & 20 & 20 & $\mathbf{7 3}$ \\
$\mathbf{4}$ & 8 & 15 & 16 & 14 & $\mathbf{5 2}$ \\
total & 8 & 18 & 19 & 23 & $\mathbf{6 8}$ \\
& $\mathbf{5 4}$ & $\mathbf{6 7}$ & $\mathbf{6 8}$ & $\mathbf{7 0}$ & $\mathbf{2 5 9}$ \\
\hline
\end{tabular}

* Apresentou uma larva aderida ao peritônio. encontrado nos grupos tratados com citronela in natura pode ser também devido ao comportamento de fixação da larva sobre a mucosa do trato gastrointestinal, uma vez que a fixação ocorre por aderência da extremidade anterior da larva à mucosa. Este fato determinaria provavelmente uma obstrução da abertura oral, dificultando a ingestão do princípio ativo da planta pelo parasito.

Outra possibilidade que justifique a discreta ação larvicida sobre Contracaecum sp., seria a concentração dos compostos geraniol e citronelol disponível na planta. Embora a quantidade de planta ingerida tenha sido a maior próxima possível de acordo com a capacidade de ingesta do coelho, ainda assim a quantidade dos compostos nesta quantidade da planta, talvez não tenha sido suficiente para a obtenção de um adequado efeito larvicida, havendo necessidade de realização de novos testes da ação larvicida e também da

Tabela 2: Larvas de Contracaecum sp., vivas aderidas ou soltas e mortas, recuperadas no período pós-infecção de coelhos dos grupos teste (GT1, GT2 e GT3) e Grupo Controle Parasitado (GCP)

\begin{tabular}{cccccccccccc}
\hline & \multicolumn{1}{c}{ LARVAS } & \multicolumn{1}{c}{ GCP } & \multicolumn{2}{c}{ GT1* } & \multicolumn{2}{c}{ GT2 } & \multicolumn{2}{c}{ GT3 } & \multicolumn{2}{c}{ Total } \\
& $\mathrm{n}$ & $\%$ & $\mathrm{n}$ & $\%$ & $\mathrm{n}$ & $\%$ & $\mathrm{n}$ & $\%$ & $\mathrm{n}$ & $\%$ \\
Mortas & 0 & 0 & 1 & 0,39 & 2 & 0,77 & 4 & 1,54 & 7 & 2,70 \\
Vivas aderidas & 35 & 13,51 & 57 & 10,42 & 54 & 20,85 & 52 & 20,08 & 198 & 76,45 \\
Vivas soltas & 19 & 7,33 & 9 & 3,47 & 12 & 4,63 & 14 & 5,40 & 54 & 20,85 \\
total & 54 & 20,85 & 67 & 25,87 & 68 & 26,25 & 70 & 27,03 & 259 & 100 \\
\hline
\end{tabular}

* Apresentou uma larva aderida ao peritônio.

\section{Discussão}

O teste da citronela in natura sobre as larvas de Contracaecum sp., não tem precedentes na literatura. No entanto, Hierro et al. (2006) testaram a ação larvicida de alguns derivados monoterpênicos in vivo, utilizando camundongos infectados com larvas de Anisakis sp., observando eficácia do extrato alcoólico de citronelol na concentração de $46,90 \mathrm{mg}$ associado a $0,5 \mathrm{ml}$ de óleo de oliva. O citronelol é um dos compostos constituintes da citronela, porém, os resultados não compatíveis podem ser explicados considerando-se as diferenças entre os organismos hospedeiros e principalmente devido às características específicas dos parasitos em teste, Anisakis sp. e Contracaecum sp. Embora pertençam à mesma família, a proximidade filogenética não necessariamente está relacionada com respostas similares de sensibilidade ao composto testado.

A ação in vitro de compostos monoterpênicos foi testada por Hierro et al. (2004), observando a ação larvicida dos compostos geraniol e citronelol. A ação larvicida destas substâncias ocorreu sobre o sistema excretor das larvas e sobre a integridade da cutícula e do trato digestivo, considerando-se que as larvas obrigatoriamente precisam ingerir o composto testado para observação deste efeito desejado. Desta forma, o baixo número de larvas mortas toxicidade aguda para coelhos, utilizando-se maiores concentrações dos compostos citronelol e geraniol.

O uso de veículos alcoólicos, aquosos ou oleosos também pode interferir na ação larvicida, por alteração da solubilidade dos compostos encontrados na planta. Trabalhos sobre potencialização do efeito de compostos antiparasitários foram feitos por Frezza et al. (2007), utilizando a incorporação de anti-helmínticos a cápsulas lipossomais. Esta técnica aumentou a disponibilidade do composto no organismo de cobaios, assim como a sua solubilidade, proporcionando uma maior absorção pelos parasitos e potencializando os danos causados a eles.

Ao avaliar os grupos pelo teste de qui-quadrado, considerando-se a proporção de helmintos inoculados e recuperados, observou-se que houve uma diferença estatisticamente significante entre o GCP e demais grupos, apenas no primeiro dia. Para os demais dias, essa diferença não foi clara $(p=0,1)$. Os resultados apresentados nas repetições dos grupos são homogêneos.

Os dados clínicos e hematológicos apresentados pelo GCC não revelaram indícios de toxicidade da planta sobre o organismo dos animais utilizados neste trabalho. Testes de toxicidade desta planta sobre o organismos de coelhos ainda não haviam sido realizados. 


\section{Referências}

ALLEN, T.C. Hematoxylin and eosin. In: Prophet EB et al., editors. Laboratory methods in histotechnology. Washington, DC: Armed Forces Institute of Pathology; p. 53-58, 1992.

AMATO, J.F.R.; BOEGER, W.P.; AMATO, S.B. Protocolos para laboratório: coleta e processamento de parasitos de pescado. Seropédica: Imprensa Universitária, UFRRJ; 1991.

BARROS, L.A.; TORTELLY, R.; PINTO, R.M.; GOMES, D.C. Effects of experimental infections with larvae of Eustrongylides ignotus Jäegerskiold, 1909 and Contracaecum multipapillatum (Drasche, 1882) Baylis, 1920 in rabbits. Arq Bras Med Vet Zootec, v. 56, n. 3, p. 325-332, 2004.

BARROS, L. A.; MORAES FILHO, J.; OLIVEIRA, R. L. Nematóides com potencial zoonótico em peixes com importância econômica provenientes do rio Cuiabá. Rev Bras Ci Vet, v. 13, n. 1, p. 55-57, 2006.

BARROS, L. A.; MORAES FILHO, J.; OLIVEIRA, R. L. Larvas de nematóides de importância zoonótica encontradas em trairas (Hoplias malabaricus Bloch, 1794) no município de Santo Antonio do Leverger, MT, Arq Bras Med Vet Zootec, v. 59, n. 2, p. 533-535, 2007.

BRASIL. Agência Nacional de Vigilância Sanitária. Portaria n. .116 , de 8 de agosto de 1996. Dispõe sobre normas para estudo da toxidade de produtos fitoterápicos. Diário Oficial da União, Poder Executivo, Brasília, DF, 10 agosto 1996.

BRASIL. Ministério da Agricultura. Instrução Normativa no. 3, de 17 de janeiro de 2000. Regulamento técnico de métodos de insensibilização para o abate humanitário de animais de açougue. S.D.A./M.A.A. Diário Oficial da União, Brasília, DF, p.14-16, 24 de janeiro de 2000, Seção I.

CHIEFFI P. P.; GORLA, M. C. O.; VIEIRA TORRES, D. M. A. G.; SOUZA DIAS R. M. D.; MANGINI, A. C. S.; MONTEIRO, A. V.; WOICIECHOVSKI, E. Human infection by Phagicola sp. (Trematoda-Heterophyidae) in the minicipality of Registro, São Paulo State, Brazil. J Med Hyg. v. 95, p. 346-348, 1992.

CHIEFFI, P. P.; LEITE, O. H.; DIAS R. M. D. S.; TORRES, D. M. A. V.; MANGINI, A. C. S. Human parasitism by Phagicola sp. (Trematoda-Heterophyidae) in Cananéia, São Paulo State, Brazil. Rev Inst Med Trop São Paulo. v. 32, p. 285-288, 1990.

DIAS, R. M.; MANGINI, A. C.; TORRES, D.; VELLOSA, S.; SILVA, R. M.; SILVA, M. I. Introdução de Clonorchis sinensis por imigrantes do leste asiático no Brasil e a suspensão da obrigatoriedade de exames laboratoriais para obtenção de vistos de permanencia. Rev. Bras Anal Clin. v. 24, n. 2, p. 2930, 1992.

EDUARDO, M.B.P.; SAMPAIO, J.L.M.; GONÇALVES, E.M.N.; CASTILHO, V. L. P.; ALBUQUERQUE, A. L. S. R.; PAVANELLO, E. I.; VIGILATO, M. A. N.; MARSIGLIA, D. A. P.; ATUI, M. B.; TORRES, D. M. A. G. V. Diphyllobothrium spp.: um parasita emergente em São Paulo, associado ao consumo de peixe cru - sushis e sashimis, São Paulo, março de 2005. Bol Epidemiol Paulista, n. 15, p. 1-5, 2005a.
EDUARDO, M.B.P.; SAMPAIO, J.L.M.; SUSUKI, E.; CÉSAR, M. L. V. S.; GONÇALVES, E.M.N.; CASTILHO, V. L. P.; ALBUQUERQUE, A. L. S. R.; PAVANELLO, E. I., VIGILATO, M. A. N.; LÍRIO, V. S.; MANTESSO, I. S.; ZENEBON, O.; MARSIGLIA, D. A. P.; ATUI, M. B.; RODRIGUES, R. S. M.; RODRIGUES, R. M. M. S.; TORRES, D. M. A. G. V.; LATORRE, W. C. Investigação epidemiológica do surto de Difilobotríase, São Paulo, Maio de 2005. Bo Epidemiol Paulista, n. 17, p. 1-12, 2005b.

EMMEL, V.E.; INAMINE, E.; SECCHI, C.; BRODT, T. C.; AMARO, M. C.; CANTARELLI, V. V.; SPALDING, S. Diphyllobothrium latum: relato de caso no Brasil. Rev. Soc. Bras. Med. Trop. v. 39, n. 1, p. 82-84, 2006.

FELDMAN, D.B; SEELY, J.C. Necropsy guide: rodents and the rabbit. New York: CRC Press Inc.; 1988.

FREZZA, T.F.; MADI, R.R.; BANIN, T.M.; PINTO, M. C.; SOUZA, L. R.; GREMIÃO, M. P. D.; ALLEGRETTI, S. M. Efeito do Praziquantel incorporado a lipossomas nos diferentes estágios de desenvolvimento dos ovos de Schistosoma mansoni. Rev. Ciênc. Farm. Básica Apl, v, 28, n. 2, p. 209214, 2007.

HIERRO, I.; VALERO, A.; PÉREZ, P.; CABO, M. M.; MONTILLA, M. P.; NAVARRO, M. C. Action of different monoterpenic compounds against Anisakis simplex s.l. $\mathrm{L}_{3}$ larvae. Phytomedicine, v. 11, n. 1, p. 77-82, 2004.

HIERRO, I.; VALERO, A.; NAVARRO, M.C. In vivo larvicidal activity of monoterpenic derivatives from aromatic plants against L3 larvae of Anisakis simplex s.I.. Phytomedicine, v. 13, n. 7, p. 527-531, 2006.

LEITE, O.H.M.; HIGAKI, Y.; SERPENTINI, S.L.P.; CARVALHO, S. A.; AMATO NETO, V.; TORRES, D. M.; DIAS, R. M.; CHIEFFI, P. P. Infecção por Clonorchis sinensis em imigrantes asiáticos no Brasil: tratamento com praziquantel. Rev Inst Med Trop São Paulo, v. 31, n. 6, p. 416-422, 1989.

MITRUKA, B.M.; RAWNSLEY, H.M. Clinical biochemical and hematological reference values in normal experimental animals. New York: Masson Publ., 1977.

PROPHET, E.B. Fixation. In: Prophet EB et al., editors. Laboratory methods in histotechnology. Washington, DC: Armed Forces Institute of Pathology; p. 29-31, 1992.

SANTOS, F.L.N.; FARO, L.B. The first confirmed case of Diphyllobothrium latum in Brazil. Mem Inst Oswaldo Cruz, v. 100, n. 6, p. 685-686, 2005.

VICENTE, J.J.; RODRIGUES, H.O.; GOMES, D.C.; PINTO, R.M.. Nematóides do Brasil. Parte IV: Nematóides de aves. Rev. Bras Zool, v. 12. p. 1-273, 1995. 